a relativistic effect, would then also be a test of Einstein's theory of gravitation.

\section{Conclusion}

It is fascinating to see how, starting with the discovery of the pulsars, many more and ever more exotic astronomical objects involving neutron stars have been found. These massive bodies of densities inaccessible in terrestrial laboratories, seem to be set up in such a variety of celestial systems that their properties can be unravelled step by step. These are good times for astrophysicists.

\section{References}

1. TRÜMPER, J., PIETSCH, W., REPPIN, C., VOGES, W., STAUBERT, R., and KENDZIORRA, E., Ap. J. Letters 1978 (in press)

2. "X-Ray Binaries" Proc. of NASA Symp. at GSFC (Maryland) (1975). This volume has been used as a standard reference for the data presented.

3. VAN DEN HEUVEL, E.P.J. and HEISE, J. Nat. Phys. Sci. 239, 1972, 67

4. TAYLOR, J.H., HULSE, R.H., FOWLER, L.A., GULLAHORN, G.E., and RANKIN, J.M., Ap. J. 206, 1976, L53

5. BÖRNER, G., Springer Tracts in Mod. Phys. 69 (1973) 1

\section{Universiteit van Amsterdam}

The group for Atomic Physics in the Zeeman Laboratorium has a vacancy for a

\section{senior research associate $(\mathrm{m} / \mathrm{f})$}

who will participate in current activities (term analysis of spectra and laser spectroscopy), with the special duty to take charge of theoretical support. Applicants should be qualified specialists in the field of theoretical atomic structure and spectroscopy and have a good knowledge of experimental methods. Educational duties include the teaching of theory of atomic spectroscopy at an advanced level and coaching of graduate students. Command of the English language is mandatory while a reasonable knowledge of Dutch is expected to be acquired during service. Applications with curriculum vitae, list of publications and names of referees to be received before July Ist, by

Dr. P. F A. Klinkenberg Zeeman Laboratorium Plantage Muidergracht 4 IOI8 TV Amsterdam - The Netherlands.

\title{
Lattice Dynamics
}

An International Conference on Lattice Dynamics was held in Paris, September 5-9, 1977, sponsored by EPS, IUPAP and SFP. The programme of this conference reflected recent developments in this field, a large part being devoted to phase transitions related to mode softening and central peak. The remarkable developments of non-linear physics have also penetrated the field of lattice dynamics : a session was devoted to solitions. Much interest seems to focus on phonon driven phenomena: superconductivity, ferroelectricity, melting and to non perfectly (ill) condensed matter, defects in materials, disordered phases, amorphous solids and liquids and materials of specific interest like superionic conductors.

Instead of an overall account of the Conference a few significant contributions in the fields of Microscopic Theory, Solitons and Electron-Phonon Interactions are summarized.

\section{Microscopic and Model Theories of Lattice Dynamics}

Microscopic calculations of phonon dispersion curves are much simpler in metals than in non-metals. The po- tential energy of electrons, in simple metals at least, is small compared to their kinetic energy: the ratio can then serve as a small parameter. The electronic response to core displacements can be described in terms of a simple complete set of equilibrium functions and yield a unified formulation of microscopic lattice dynamics both in metals and non-metals. A rigorous "first-principle" calculation has yet to be performed. The effort in this direction is nevertheless interesting for at least two reasons: microscopic theory should help us understand in detail how the dynamical spectrum of solids arises from their crystal and electronic structures employing Fermi surface effects, covalent, ionic, metallic binding, etc. The second reason is that such theory should connect the same macroscopic physics to related properties such as superconductivity, crystallographic phase transitions, optical and transport properties and the like.

S.K. Sinha 1) advocates the approach using the density response function of the electron system. With the formulation using this approach, he examines the occurrence of phonon soft-

\section{Balkanski, Paris}

(Chairman, Condensed Matter Division)

ening and lattice instabilities in d-band metals pointing out a strong correlation between such behaviour and a high density of states at the Fermi level associated with the d-states, and also with the occurrence of relatively high $T_{c}$ in superconductivity. Model calculations considering several quasi-degenerate d-bands at the Fermi level yields, for $\mathrm{Nb}$, the characteristic dips in the longitudinal branches along [100] and [111]. The dip along [111] is probably related to the "central peak" observed in $\mathrm{Nb}$ and $\mathrm{Nb}-\mathrm{Zr}$ alloys. $\mathrm{NbC}$ and $\mathrm{TaC}$ show also pronounced softening type anomalies in the acoustic modes reproduced well by the same model. It has also been pointed out that the response function $\mathrm{X}_{\mathrm{c}}(\vec{q})$, peaks in $\mathrm{NbC}$ due to Fermi surface nesting features, which occur also at the position of the anomalies. This may indicate correlation between the electron-phonon matrix elements and the electronic structure.

A similar coincidence with regard to peaks in $X_{\mathrm{c}}(\vec{q})$ and the position in $q$ of the maximum softening, is mentioned in the "charge-density-wave" materials, e.g. the layered transition 
metal di-chalcogenides and in rutilestructure transition-metal oxides, $\mathrm{NbO}_{2}$ and $\mathrm{VO}_{2}$. For these materials it is also observed that the phonon at the wave vector of the instability does not actually soften to zero frequency, but instead, in the neutron scattering a central component appears and grows as the instability temperature is approached.

Introducing for low frequencies a Debye-like relaxation for the density fluctuations of the non interacting system, one obtains a three-peaked structure with "phonon sidebands" and a central component.

R. Zeyher has reviewed ${ }^{2}$ ) microscopic theories of lattice dynamics which use localized non-orthogonal nonequilibrium wave functions for the occupied states. The microscopic expression for the non-equilibrium cohesive energy is compared with the corresponding expressions of the two most well-known and successful phenomenological models: the shell and the deformation dipole models. The two models are not equivalent and a challenging problem is the quantum mechanical justification of these models.

Zeyher's own work has shown that a microscopic theory based on Adams-Gilbert orbitals leads to a slightly more general energy expression in ionic crystals than the simple shell model, and to good first principle phonon dispersion in LiD. It has also been shown that the microscopic expression is only slightly more general than that of the deformation dipole model.

\section{Solitons}

The enormous emphasis hiven to non-linear physics in all fields is also pertinent to lattice dynamics. A.R. Bishop treats the topic of non-linear equations in physics, specifically those supporting "particle-or pulse-like" travelling wave solutions. He recognizes that these equations have been familiar in various contexts for many decades but have enjoyed a remarkable revival of interest in the past few years, stimulated by concurrent developments in mathematics, condensed matter physics, statistical mechanics and quantum field theory. The most important development is the recognition that certain types of non-linear equation can be handled without the conventional recourse to linearization procedures, and that fully non-linear solutions exist which cannot be reached by finite order linear perturbation theory, but which can have highly distinctive physical consequences. The conviction of Bishop. based on the universal recognition of this fact, is that the non-linear techniques and concepts should become tools of the whole physics community, much as linearization and (linear) normal modes are already.

Non-linearity is an essential aspect of critical phenomena at many levels. Using real space configuration, Bishop describes models where strongly nonlinear excitations are important energetic and dynamic considerations, and can dominate static and dynamic critical behaviour.

The typical equation is a wave equation combining elements of nonlinearity and dispersion, which can balance dispersive and self-steepening tendencies to support a "solitary wave", i.e., a spatially limited travelling wave that retains its shape while propagating. This equation should admit large amplitude "kink" or solitary wave solutions of velocity $V$ where the field varies between neighbouring potential minimum values over an essentially localized region. The solitary wave has a particle-like behaviour. Compared to the linear normal modes (phonons, magnons) which are approximately independent in wavevector space, the solitary waves (solitons and anti-solitons) are much more nearly independent in real space. One also distinguishes the breather modes which correspond to bound solitonantisoliton pairs.

The non-linear equations such as the "sine-Gordon" equation are extraordinarily ubiquitous, with common ingredients of wave propagation in the presence of a "pinning", "locking" or "registering" potential applying to dislocations, ferromagnets, Josephson phase-locking, pinning in charge density waves, commensurate to incommensurate phase transitions, superionic conductors, rotational phase transitions in molecular crystals, structures in surface deposits, etc.

Much of the analytic progress made is restricted to one dimension which has indeed some very special properties.

In a general sense, the non-linear excitations should be viewed as "order parameter defect structures". In one dimension and with the order parameter having only one component, the only type of defect profile is a domain wall. The variety of situations where walls occur is large but for dimensions equal or higher than two, and number of components of the order parameter $n \geqslant 2$ a far richer variety of defect types is possible.

Bishop also points out that solitonbearing equations are bare descrip- tions of a great variety of problems and in view of the remarkable mathematical information available for them, they offer rare soluble systems. Hence their study is quite relevant and useful if realistic physical constraints are judiciously imposed.

\section{Electron-Phonon Interactions}

Electron-phonon interactions were the central point of discussion at the Conference. It is the main feature in microscopic theories, the keystone in phase transitions and of essential concern in resonant light scattering processes. Two cases are presented : the resonant interactions of acoustic phonons with excitonic polaritons, and the first order spectrum replicas in alloys. The first is a beautiful demonstration of a theoretical prediction, the second a recent discovery.

Brenig, Zeyher and Birman have shown in a theory of Brillouin Scattering by excitonic polaritons in semiconductors, that one should observe, instead of the usual Brillouin doublet, an octuplet in the energy region above the longitudinal exciton energy $E_{L}$ where two distinct polariton waves can propagate in the crystal. A resonant enhancement is predicted to occur when the ingoing or outgoing photons are resonant with the 1 s exciton level.

Two groups have performed experiments which largely confirm these theoretical predictions: Claude Weisbuch and Rainer G. Ulbrich have worked on $\mathrm{GaAs}^{4}$ ) and $\mathrm{G}$. Winterling and E.S. Koteles on CdS ${ }^{5}$.

With the experiments carried out on GaAs by Weisbuch and Ulbrich the following pertinent observations are reported :

(i) Polaritons are predominantly coupled to LA-phonons. The observation of the Brillouin shifts well below and above the longitudinal exciton energy $E_{\mathrm{L}}$. where the polariton dispersion curve is essentially photonlike, permits the velocity of the phonons interacting with the polaritons, to be measured.

(ii) The Brilliouin lines are broadened due to the high absorption coefficient $\alpha$ in the polariton region. Light penetrates layer only $\approx \omega^{-1}$ thick and from the uncertainty principle, the emitted or absorbed phonons have a certain dispersion $k$. The maximum relative broadening occurs at the maximum absorption. This is the case when the ingoing polariton is at resonance and has pentration depth $\mathrm{c}^{-1}$ or when the outgoing polariton is resonant.

(iii) Over the expected smoothly resonant variation, one observes an 
anti-resonant dip at the maximum of polariton absorption: the decrease of efficiency indicates that re-absorption effects at exact resonance prevail over the probability enhancement.

(iv) Resonant increase and cooling of polariton fluorescence have been observed and connected with Resonant Brillouin Scattering. In a backscattering configuration, one observes polariton escaping from the crystal with a wave vector opposite to that of the exciting light. Polaritons scattered by one longitudinal acoustic phonon give rise to the well defined Resonant Brillouin Scattering peaks. All other polaritons, having suffered several successive processes are smoothly distributed and give rise to Resonant Polariton Fluorescence. Thus the Resonant Brillouin Scattering involves a memory of the initial state, whereas the Resonant Polariton Scattering originates from a randomised distribution of more or less thermalized phonons.

The experiments carried out on CdS by Winterling and Koteles have determined the dispersion of the exciton-polariton and have led also to the study of the properties of high frequency acoustc phonons and the interaction between phonons and polaritons.

Below the longitudinal exciton energy $E_{L}$ "allowed" longitudinal acoustic phonon and "forbidden" transverse acoustic phonon scattering processes are observed, whose line shifts fit well with the two-branch model of the polariton dispersion.

Above $E_{L}$ a set of narrow strong lines is observed which cannot be explained by the predicted one phonon process based on the two-branch model.

The observation of a new resonance phenomenum was also reported by $M$. Balkanski, C. Hirlimann and J.F. Morhange: replicas of the first-order phonon spectrum ${ }^{6}$ ). The first-order Raman spectrum of mixed III-V semiconductor compounds contains the characteristic normal modes of the two compounds. At resonance conditions, phonon replicas of this spectrum are observed, only in disordered system; the first-order spectrum is entirely reproduced, shifted by the frequency of the longitudinal optical mode. The scattered intensity of a replica can be even higher than that of the first order spectrum depending on the frequency of the incident beam. Such replicas are absent from the spectra of pure crystals.

Resonance occurs at a photon energy close to the band gap. Replicas have been observed also for higherorder LO phonon combinations. These results are interpreted by a model in which the relevant resonant intermediate state is a localized exciton strongly interacting with the LOphonon. The different replica orders correspond to resonance states formed by differently "dressed" trapped excitons.

It is obvious that the choice presented here is personal and purposely limited to only very few of the many high-level, timely and important contributions presented at this very stimulating scientific meeting.

\section{References}

References are to the Proceedings of the International Conference on Lattice Dynamics (Flammarion Sciences, Paris), 1978.

\section{New President of EPS, Antonino Zichichi}

Antonino Zichichi, a founding member of EPS, is a high energy physicist, presently engaged on a systematic study of the proton structure at the CERN machines ISR and SPS. Professor of Advanced Physics at the University of Bologna (Italy) he was the Director of the Postdoctoral School of Physics at that University. Zichichi is well known in the international physics community; the study of lepton pairs produced in hadronic interactions, the search of heavy leptons produced in $\mathrm{e}^{+} \mathrm{e}^{-}$annilations, exemplify his pioneering contributions in two branches of sub-nuclear physics where a decade was to pass before the phenomena were understood. His research activity covers many fields: the high precision tests of Quantum Electrodynamics, the systematic study of the electromagnetic form factors of the proton and the pseudscalar mesons, the first measurements of the $\left(w_{-} \varnothing\right)$ mixing angle, the discovery of the antideuteron and of the $2 \delta$ decay of the $X$ meson, the high precision measurement of the weak coupling constant...

$\mathrm{He}$ is the founder and the Director of the "Ettore Majorana" Centre for Scientific Culture, established in 1962 and now consisting of 70 national and international Schools - the same Cen- tre which has put a considerable number of places at the disposal of EPS and has offered advantageous terms to Divisions wishing to hold conferences there. In June 1977 Zichichi was elected President of the italian Istituto Nazionale di Fisica Nucleare (INFN) which promotes fundamental research in sub-nuclear and

nuclear physics. More recently, at the plenary meeting of the European Committee for Future Accelerators, he was elected chairman of the working committee studying the project for a large electron-positron colliding beam device (LEP) that ECFA considers is the European high energy machine for the 1980s.

\section{LASER DEPARTMENT \\ INSTITUTE OF APPLIED PHYSICS, UNIVERSITY OF BERN}

Applications are invited for the position of :

\section{RESEARCH PROJECT LEADER}

Qualifications: Essential - Experience in Gas Laser Physics with preferably a knowledge of electronic transition lasers and related discharge techniques.

Desirable - Some knowledge of German.

The position carries responsibility for the Gas-Laser group (staff complement : $2 \mathrm{PhD}, 1$ dipl. and $1 \mathrm{PhD}$ candidate) which is part of the Laser Department (staff complement : 25).

The Gas-Laser group is presently working on CW high power lasens in the visible and near UV and on pulsed TEA discharges.

Equipment available for studies is the following :

- TEA discharge system for metal vapours

- Powerful UV sources (coherent and incoherent), pulsed and CW dye lasers

- $300 \mathrm{~kW}$ DC power supply (600 A or $1 \mathrm{kV}$ )

Additional information may be obtained trom, and applications accompanied by curricula vitae sent to: Prof. Dr. Heinz. P. Weber

institute of Applied Physics, University of Bern

Sidlerstrasse 5

CH-3012 Bern

The position will be open from 1 July, 1978. 